\title{
Autorité ET AUCTORIALITÉ AU SEIN DE GROUPES WHATSAPP CHRÉTIENS LIBANAIS
}

\author{
Rosemary ISKANDAR ${ }^{1}$
}

\begin{abstract}
Les groupes WhatsApp sont utilisés par des chrétiens libanais pour partager des prières et une foi commune, entre identité et culture. Mais s'y jouent également des enjeux d'autorité (cléricale et laïque) et de pouvoir mais aussi de propriété intellectuelle (droit d'auteur, auctorialité) qu'une observation non participante et des entretiens nous permettent d'analyser, dans la formation de l'espace religieux qu'active le groupe. Le régime techno-discursif de WhatsApp permet en effet, en contexte chrétien, de donner une nouvelle place aux laïcs, tout comme il permet aux prêtres d'accroître leur audience spirituelle.
\end{abstract}

L'interactivité et la créativité des utilisateurs sur internet ont modifié la présence religieuse en ligne (Campbell, 2012). Une possibilité nouvelle d'interaction entre les religionnaires (Favret-Saada, 2017) en présence virtuelle apparaît, notamment au sein des groupes numériquement animés (Jonveaux, 2013, p. 117). Ainsi, des contenus spirituels sont-ils proposés désormais en ligne (Catellani, 2014) : des prières sont partagées par des croyants sur WhatsApp et consultées par d'autres membres qui, sans doute, ainsi, ravivent leur foi, l'entretiennent de nouveaux discours et de nouvelles images, puisant dans un même fond de

1 Rosemary IsKandaR est membre du Laboratoire de Recherches en Sciences de l'Information et de la Communication (SIC.Lab Méditerranée), Université Côte d'Azur, Nice.

Recherches en communication, $\mathrm{n}^{\circ} 53$ - Article publié le 15/09/2021 
« tradition », sans cesse réactivée. Les catholiques (maronites) libanais, qu'ils soient religieux, prêtres ou laïcs, se rencontrent aujourd'hui sur des réseaux sociaux, et plus particulièrement via WhatsApp, application très prégnante au Liban, et partagent des prières et des informations à portée identitaire qui les intéressent. Nous menons depuis plusieurs années une recherche sur ces groupes chrétiens qui se constituent sur WhatsApp. Ce sont ces espaces d'échange que nous proposons d'examiner dans cet article, en considérant particulièrement les questions liées aux jeux d'autorité et d'auctorialité, liant pouvoir et prière.

La problématique de recherche de cet article s'articule autour de deux questions. Tout d'abord, il s'agit de comprendre comment les échanges de prière et le partage de l'espace du groupe religieux virtuel s'effectuent pour les administrateurs des groupes WhatsApp. Ensuite, comment l'autorité d'un administrateur et le rôle d'un prêtre sont interprétés dans un groupe. Autrement dit, nous nous intéresserons ici à l'administration spirituelle et technique de ces groupes, et aux enjeux de pouvoir qui les parcourent, entre autorité et auctorialité des ressources spirituelles proposées, au cœur de l'espace orant chrétien numérisé.

\section{Contexte et présentation du terrain de recherche}

La présence de l'Église catholique sur internet est pensée par les papes depuis Jean-Paul II, et mise en œuvre tant par des conférences épiscopales, des diocèses, des paroisses que des ordres religieux et des mouvements spirituels, sous l'égide de clercs ou de laïcs, dans différents pays. En outre, le pape François a insisté sur l'importance du rôle des laïcs dans la vie de l'Église catholique et dans l'évangélisation (Forestier \& Waymel, 2018). Parmi les nombreux dispositifs numériques utilisés dans le cadre de la vie chrétienne en ligne (sites web, pages et groupes Facebook, Twitter, YouTube, Instagram...), WhatsApp connaît un certain succès au Liban dans la vie et l'expression chrétiennes, et de nombreux laïcs y contribuent.

\subsection{Le Liban : contexte religieux}

Le Liban étant un pays multiconfessionnel, la religion y est très présente dans les pratiques culturelles et sociopolitiques. En effet, ce pays possède une diversité de confessions religieuses dans plusieurs régions, il est considéré comme l'un des pays les plus diversifiés sur le plan religieux au Moyen-Orient. Effectivement, il existe 18 confessions 
religieuses reconnues officiellement par l'État libanais, dont 4 groupes musulmans et 12 groupes de chrétiens avec le peuple juif et les druzes. Ce pays est composé de 33,7\% de chrétiens (les catholiques maronites sont le plus grand groupe chrétien), $61,1 \%$ de musulmans, $5,2 \%$ de druzes et de diverses autres minorités religieuses, y compris les juifs ${ }^{2}$. Cependant, les druzes sont considérés comme des musulmans dans la répartition du pouvoir politique dans le pays.

On distingue plusieurs types de chrétiens au Liban: les maronites, les melkites, les Grecs orthodoxes, les syriaques orthodoxes et catholiques, les Arméniens orthodoxes et catholiques, les évangéliques. D'ailleurs, les chrétiens maronites constituent la plus importante communauté chrétienne du Liban où siège l'Église maronite, une des Églises catholiques orientales, qui est toujours unie à Rome.

Sur le plan du contexte confessionnel, les chrétiens libanais peuvent être amenés à se penser comme une minorité fragilisée. En effet, si les Arabes chrétiens du Proche-Orient étaient autrefois majoritaires (Boëdec, 2006), aujourd'hui tous les gouvernements arabes de la région, à l'exception du Liban, ont inscrit la charia (la loi islamique) dans leur constitution comme source principale du droit (Ibid.). Cependant, dans le monde arabe, le Liban est le seul pays qui a un président chrétien (par coutume constitutionnelle). La population libanaise est fortement caractérisée par des appartenances religieuses et communautaires. Ainsi, les « chrétiens » vivaient dans des espaces " chrétiens » et les « musulmans » dans leurs espaces propres. D'ailleurs, plusieurs municipalités interdisent formellement aujourd'hui les ventes immobilières entre chrétiens et musulmans (Jreijiry, 2018). Le Liban est considéré comme un pays où les chrétiens restent très présents, si on le compare avec les autres pays arabes.

\subsection{Terrain de recherche et positionnement du chercheur}

Depuis trois ans $\mathrm{s}^{3}$, nous menons une observation des échanges dans les groupes WhatsApp chrétiens libanais entre les participants chrétiens arabes. Nous retenons une partie du travail ethnographique réalisé sur

2 Central Intelligence Agency. World factbook: Lebanon. Informations trouvées sur : https:// www.cia.gov/library/publications/the-world-factbook/geos/le.html Consulté le 22 avril 2020

3 La présente recherche a débuté en août 2017 et s'achève pour cet article en août 2020. Nous tenons à remercier David Douyère (Prim, Université de Tours) pour sa relecture attentive de cet article. 
ces terrains de recherche en vue d'examiner plus particulièrement, en premier lieu, la façon dont la présentation du groupe est pensée par les administrateurs (en considérant, ici, deux groupes). Nous observons ensuite la façon dont l'autorité est présente et vécue dans un groupe spécifique, en examinant comment les prières sont proposées par les croyants qui échangent en ligne, soulevant des questions d'auctorialité et de propriété intellectuelle. Pour ce faire, nous nous appuierons sur des observations et des entretiens, rassemblés ici sous formes de cas, centrés autour des administrateurs de ces groupes et des créateurs de prières. ${ }^{4}$.

Notre position de chercheuse par rapport à ce terrain peut être identifiée et spécifiée de façon suivante : nous sommes culturellement chrétienne maronite, mais nous ne participons à aucun groupe religieux en ligne, et notamment sur WhatsApp. Il y a donc une proximité culturelle dans le langage et les références, mais une distance en termes de pratique religieuse, qui offre ici à la chercheuse à la fois un espace de compréhension et d'interrogation distanciées. C'est par une conversation ordinaire de la vie sociale, au Liban, en 2017, que notre attention a été portée sur les groupes de prière sur WhatsApp. C'est ainsi que nous avons ensuite pu intégrer les premiers groupes chrétiens. Au fur et à mesure, des liens permettant l'intégration dans d'autres groupes de prière ont été partagés dans la discussion dans la communauté virtuelle de prière. C'est ainsi que nous avons pu intégrer le groupe de Léna, les groupes de Sylvie et ceux du prêtre Jean.

Léna est pharmacienne, croyante catholique et célibataire d'une quarantaine d'années ${ }^{5}$. Elle fait partie de " l'Équipe spirituelle, la Trinité » créée en 1986 par un prêtre libanais ; cette équipe est un groupe catholique qui s'adresse aux Arabes catholiques. Avant d'ouvrir son groupe sur WhatsApp, Léna a demandé une autorisation à son accompagnateur spirituel. Celui-ci fait partie du groupe comme observateur et vérifie les données postées sur WhatsApp. De 2016 à 2019, Léna a pu élargir sa communauté en ligne et elle a créé sept groupes qui ont tous le même nom et qui sont numérotés de 1 à 7 . Ces groupes diffusent les mêmes prières sur WhatsApp et comportaient en mai 2019 environ

4 Les prénoms ont été modifiés pour des raisons de confidentialité.

5 Nous avons mené un entretien avec Léna en avril 2019. L'observation non participante de son groupe WhatsApp « Médite et prie » a commencé en août 2017. 
1600 personnes. Le prêtre maronite Jean ${ }^{6}$ est administrateur et créateur de sept groupes sur WhatsApp qui portent sur différents sujets mais qui traitent tous du christianisme.

Deux autres maronites ont fait également l'objet d'entretiens après la réception de leur prière sur WhatsApp. Sylvie, qui appartient à cinq groupes que nous suivons, reprend des prières et les modifie en supprimant le nom ou l'institut auteur de la prière, avant de les diffuser dans les groupes qu'elle gère en propre. Pierre, un chrétien charismatique maronite, nous envoie quotidiennement des prières sur WhatsApp depuis 2016 ; il n'appartient pas à un groupe mais diffuse ses prières par une liste de diffusion via WhatsApp. Enfin, en juillet 2019, nous avons également mené un entretien avec Nabil, un Soudanais, pour saisir comment et pourquoi il nous avait « ajouté » dans sa communauté et afin de comprendre le but et l'objectif du fonctionnement de son groupe. Nous avons là quelques acteurs de ces communautés de partage de foi et de prière que sont ces groupes WhatsApp chrétiens libanais.

\section{Le groupe WhatsApp, un espace religieux communau- taire}

\subsection{Groupe religieux WhatsApp}

Sur les 20 groupes que nous avons étudiés entre 2017 et 2020, 13 contiennent entre 20 et 250 membres. Les groupes s'animent principalement de prières partagées, entre intentions de prière et textes d'oraisons partagées, parfois d'images de dévotion. Leur « community manager », si l'on peut dire, joue un rôle qui nous paraît très prégnant.

Pour rentrer dans la pratique d'animation d'un groupe, regardons, en premier lieu, les pratiques numériques d'une animatrice de groupes WhatsApp, Léna. Léna enregistre les versets bibliques qui sont envoyés non seulement dans ses groupes mais qui sont aussi partagés par certains membres avec d'autres groupes sur WhatsApp. Léna a commencé à enregistrer les versets bibliques avant tout pour les personnes qui n'ont pas le temps de lire. Interrogée sur la façon dont elle éprouve ses responsabilités envers ses groupes, elle répond, en se référant à l'exemple

6 Le prêtre Jean nous a accordé un entretien en avril puis en octobre 2019. Nous observons ses groupes (Le fond de la parole, À vrai dire, Dialogue spirituel audacieux, Sur notre intention, Activités spirituelles sur les traces de Marie, La prière de la galanterie maronite) depuis novembre 2017. 
évangélique de « la parabole des talents » (Luc 19,11) : «Les groupes sont mes mines et je dois m'occuper de ces mines », comme s'il était attendu par son Dieu qu'elle les fasse fructifier.

\subsection{Groupe : espace sacré}

Un groupe WhatsApp se caractérise par son nom, une photo et une description. Léna choisit l'icône du groupe très soigneusement car, selon elle, « le groupe est une place sacrée, et la place sacrée empêche les gens de pécher. » Léna considère que la photo qui forme l'icône (au sens numérique du terme) de son groupe est importante. À partir du moment où la personne est présente dans son groupe et une fois qu'elle accède à l'application WhatsApp, elle nous dit que " cette personne pourra identifier le groupe grâce à son icône, ainsi un pouvoir spirituel peut dominer sur sa pensée. Et par exemple si cette personne était fầchée au moment où elle a vu le groupe, elle pourrait penser à Dieu et par la suite éviter de pécher. Car l'icône du groupe, qui est une image religieuse de Jésus ou de la croix, peut calmer la personne et l'aider à trouver la paix ». L'animatrice développe donc une conscience de l'efficience spirituelle de l'icône (au double sens du terme, en réalité) choisie pour caractériser et identifier son groupe. Ce dernier a donc bien une fonction religieuse. Cette mobilisation de l'image de piété dans un dispositif numérique (Douyère, 2014) s'inscrit dans la tradition de «l'Église catholique [qui] a toujours encouragé la production d'images, d'abord pour des motifs pastoraux, pour toucher le plus grand nombre. Mais le risque de l'idolâtrie est toujours présent : l'image ne doit rester qu'un support pour la dévotion» (Lapierre et al. 2015, p. 129).

\subsection{Groupe : église et corps du Christ}

Considérons un deuxième élément de notre corpus WhatsApp. Nabil, un Soudanais, nous a ajouté dans son groupe de prière sur WhatsApp intitulé : «Qu'il est doux pour des frères de demeurer ensemble » (Psaume 133, 1). À chacune de nos questions et demande d'échange en juillet 2019, il répond exclusivement par un verset biblique, voire plusieurs, tirés du Nouveau Testament. Après quelques jours, il raconte enfin comment le groupe a commencé : « Suite à la visite des reliques de saint Marc dans notre église, j'ai partagé l'information avec mes connaissances sur WhatsApp et c'est comme ça que le groupe a commencé. Et c'est par la grâce de Dieu que j'ai pu continuer et 
progresser en servant mes frères avec les enseignements de l'Église et de la Bible. » À la question de savoir comment il a pu élargir le groupe, il répond : « Nous et vous... membres l'un de l'autre... Avec nos différentes patries, nous espérons nous rencontrer ici... et nous retrouver dans la voûte céleste... Amen. » Il ajoute, « Croyez-moi... Vous faites partie de mon corps... et nous sommes tous du Christ. Croyez-moi... si quelqu'un quitte le groupe, je perds un membre de mon corps ». Une ecclésiologie du groupe WhatsApp se forme ainsi, comparable à la conception paulinienne et catholique de l'Église, corps du Christ. Il illustre cette position d'un nouveau verset : " De même, bien que nous soyons nombreux, nous formons un seul corps dans l'union avec le Christ et nous sommes tous unis les uns aux autres comme les parties d'un même corps. » (Romains 12,5). Si Nabil considère le groupe comme le corps du Christ, alors le groupe est une partie de l'Église parce que « l'Église est le corps du Christ» (1 Corinthiens 12). Si l'Église est l'ensemble des croyants alors le groupe est aussi l'ensemble des croyants. Dans ce contexte nous pouvons dire que digitaliser l'Église dans un but de réunir tout le monde virtuellement via le numérique est considéré comme un moyen plus aisé que de produire ce rassemblement physiquement dans un lieu de culte.

\subsection{La pratique religieuse en ligne}

Dans les cas que nous étudions, l'internet donne manifestement au groupe virtuel des croyants un espace pour une pratique religieuse en ligne. Isabelle Jonveaux dans Dieu en ligne montre comment l'internet permet de prolonger l'émotion religieuse et le sentiment d'appartenance en créant un lieu de reproduction de ce lien. Les réseaux sociaux peuvent augmenter le sentiment d'appartenance religieuse, et surtout le rendre visible: "L'internet est naturellement un lieu privilégié de la mise en scène de la vie religieuse, mais cette dernière doit pour cela utiliser la grammaire de communication attachée à ce média » (Jonveaux, 2013, p. 149).

La constitution d'un espace religieux en ligne s'inscrit dans une tradition qui assigne à des lieux un sens spirituel (Salatko, 2016). Dans son article « Lieux sacrés et espace ecclésial $\left(\mathrm{IX}^{\mathrm{e}}-\mathrm{XV}^{\mathrm{e}}\right.$ siècle) 》, Antoine Brix rappelle ce que sont les lieux sacrés du christianisme, en commençant par le chœur ; l'église dans son ensemble est le deuxième lieu sacré, enfin, le monastère forme le troisième lieu sacré (Brix, 2014). L'espace sacré, selon David Chidester et Edward T. Linenthal, est d'abord un 
« espace rituel, un lieu de représentations symboliques formalisées et répétables » (Chidester et al., 1995, p. 10). «En tant qu'espace sacré, un site rituel se trouve mis à part dans un environnement "ordinaire" pour fournir une arène pour l'exécution de modèles d'actions contrôlées et "extraordinaires". Dans cette tension entre un lieu ritualisé extraordinaire et un espace ordinaire, il y a une dialectique observable dans le rôle du rituel dans la production de l'espace sacré. Les actes rituels de culte, de sacrifice, de prière, de méditation, de pèlerinage et de cérémonie consacrent l'espace sacré » (Ibid., pp. 11-12, 14). Il est clair que les lieux de culte, tels que les églises, les synagogues, les mosquées et les temples, ont été délimités, ritualisés et interprétés comme des sites spécifiques de l'espace sacré (Ibid., p. 15) : peut-il en aller de même de groupes WhatsApp?

\section{Poster des prières sur WhatsApp}

\subsection{L'édition des prières}

Au-delà de la présentation et de la proposition d'une icône qui vient représenter et qualifier, sinon sanctifier le groupe, ou du moins orienter son action, l'espace religieux se forme également de la production d'un discours, celui, codifié mais pourvu de quelque liberté, de la prière. Considérons les groupes WhatsApp animés par Sylvie. Sylvie, femme au foyer de 47 ans, est administratrice dans plusieurs groupes actifs et elle diffuse tous les jours des prières au sein de ces groupes. Comment se forme le travail éditorial qu'elle opère ? C'est d'abord dans des pages Facebook qu'elle cherche des prières qui la touchent et peuvent ainsi, estime-t-elle, toucher d'autres personnes. Elle essaie d'envoyer dans les groupes des prières courtes et faciles pour les lecteurs durant la journée. Au départ, elle envoyait des messages individuels sur WhatsApp avant de former des groupes, puis des personnes lui ont proposé de rentrer dans certains groupes. Ainsi, si le groupe est restreint, elle envoie les prières au créateur afin qu'il diffuse ses messages, et c'est en agissant de la sorte qu'elle a su gagner la confiance des administrateurs et a été promue comme administratrice de chaque groupe. Elle est aujourd'hui présente dans quinze groupes différents. Elle envoie les mêmes prières à treize groupes et pour les deux autres elle n'envoie que sa propre prière écrite avec ses mots, appelée " le pain quotidien ». Elle évite d'envoyer des vidéos et des images afin de ne pas gêner les membres par la consommation de leur forfait réseau et 
par l'espace de mémoire que peut occuper une vidéo dans un téléphone. Elle essaie aussi d'envoyer des prières courtes. La production et la diffusion s'adaptent ainsi à une considération des modalités techniques de l'usage.

La majorité des références de Sylvie proviennent de Facebook. Elle s'approprie les prières des autres en supprimant le nom de la personne ou de l'institution qui a créé ces prières. De ce fait, elle modifie aussi des prières selon ce que l'Esprit Saint, estime-t-elle, lui donne comme idée. Elle considère que les gens n'ont pas besoin de savoir d'où les prières viennent et qui les a créées car, ce qui compte le plus, à ses yeux, c'est la prière elle-même. Aujourd'hui, elle dispose d'archives de prières plagiées sur le travail d'autres personnes. Malgré l'appropriation du travail d'autres croyants, elle n'a pas été inquiétée par ces derniers. Elle explique ainsi sa démarche : «Je lis les prières qui sont partagées sur Facebook et si une phrase dans le paragraphe que je suis en train de le lire m'a plu, une phrase qui a attiré mon attention et j'ai senti cette phrase par l'Esprit Saint en moi, je fais confiance à l'Esprit Saint, autrement dit quand, en lisant, je sens que la phrase a touché mon esprit, je la prends et je l'envoie dans les groupes ».

Si Sylvie a bien participé à des réunions de prière du renouveau charismatique catholique, elle ne se présente cependant pas comme membre de ce courant spirituel, bien qu'elle utilise des expressions qui peuvent conduire à l'identifier ainsi, comme par exemple l'expression « être touché par l'Esprit Saint ». "L'expérience d'être touché par l'Esprit », relève en effet De Witte (2011), « constitue la base de la subjectivité charismatique et pentecôtiste, né de nouveau ou régénéré. Une grande partie de la pratique religieuse charismatique vise à réaliser des expériences et engage pleinement le corps comme moyen d'être "en contact" avec une présence divine » (De Witte, 2011, p. 491).

Elle ne note pas la référence de la prière : « Non je n'écris pas la référence car cela ne me concerne pas, mon but n'est pas de faire la publicité de la référence. Ce qui m'intéresse c'est de diffuser la parole qui, du fait même qu'elle m'a touché, peut toucher d'autres lecteurs. Au-delà, cela ne m'intéresse pas... » Sylvie s'est cependant trouvée une fois sollicitée par un prêtre pour avoir supprimé le nom de celui-ci d'une prière et avoir ensuite diffusé celle-ci dans les groupes : « Et il n'a pas apprécié. » Selon elle, ce prêtre entendait se mettre en avant et montrer son nom plus que de prêcher.

À rebours de ces pratiques, le prêtre maronite Jean poste dans ses groupes et sur son statut WhatsApp quasiment chaque jour deux prières 
en format image, une prière du matin et une prière du soir, prières qu'il signe de son nom en invitant à prier pour lui-même. Sa prière est caractérisée soit par un verset biblique soit par une phrase d'un saint catholique. Il raconte avoir constaté que l'on a parfois ôté son nom d'une prière diffusée. Il a contacté et questionné les diffuseurs à ce sujet car, pour lui, « c'est du plagiat et ils n'ont pas le droit de faire cela ». Outre ses prières, le prêtre Jean partage quotidiennement des passages de la Bible enregistrés par sa propre voix en mentionnant son nom à la fin de l'enregistrement.

\subsection{La conception d'une prière selon un chrétien charismatique}

Pierre $^{7}$ (âgé d'environ 40 ans), quant à lui, personnalise depuis plusieurs années une prière quasiment tous les jours et la diffuse auprès de ses proches et amis. Il est animé d'un désir de partage de sa foi : «J'étais une personne dans les ténèbres et qui a découvert la lumière. » Il dit avoir découvert « l'amour du Seigneur » et qu'il entend « transmettre la joie qu'il a ressentie » à autrui : " c'est pour cela que j'ai eu l'idée, "par l'Esprit Saint", d'évangéliser de cette manière ». Au départ la diffusion de ses prières se faisait via le courrier électronique, puis par Facebook. Avec l'apparition de WhatsApp au Liban, Pierre a commencé à évangéliser par cette application qui est la plus utilisée par les Libanais. Il rédige ses propres prières et ajoute désormais son nom car une personne lui a reproché un jour d'avoir « volé des prières »... Aujourd'hui, il envoie ses prières à presque 300 personnes, qui à leur tour, les transfèrent à leurs proches ou dans les groupes sur WhatsApp. Ses prières sont aussi placées comme statut sur WhatsApp. Il n'anime pas de groupe, car, selon lui, les groupes peuvent prendre beaucoup de temps de discussion et les actions au sein du groupe ne sont pas importantes pour prêcher. Selon lui, « le fait de prêcher dans un groupe n'est pas important. Je possède une liste de diffusion sur WhatsApp. Comme ça je prends en considération tous les types de personnes, des hommes et des femmes actifs de toutes catégories sociales ».

Le but de la prière du matin est que la personne puisse la lire le plus rapidement possible et «qu'elle porte de la puissance au profit de l'autre » dit-il. Et il ajoute : "Ces prières sont faites après que $\mathrm{j}$ 'aie prié. Dieu me donne des idées, il me parle. Souvent je lis quelque chose et Dieu me montre un point intéressant. Et je le diffuse en mettant la

7 Pierre nous a accordé des entretiens en avril, en janvier puis en mai 2020. 
mention "copié" en bas de la prière. " Ainsi, par la voix de Dieu et les pensées de Pierre, Dieu communique avec le destinataire.

À ce propos, Dein et Cook (2015) observent que « les personnes qui croient en Dieu croient que Dieu (ou les dieux) a communiqué avec les Hommes dans et par les écritures, dans l'histoire, dans l'ordre naturel, par l'intermédiaire d'autres personnes et dans une variété d'expériences qui peuvent être plus ou moins personnelles ». Il existe néanmoins des différences relatives dans l'expérience de la « voix de Dieu », qu'elle soit intérieure à l'esprit ou présente dans le monde, qui peuvent résulter de différentes manières de penser (Luhrmann, 2017, p. 133).

Pierre essaie toujours de créer une prière complète. Quant à la longueur du message, il faut que la prière soit courte afin que le lecteur daigne la lire. Il nous explique l'esprit qui prévaut à sa conception : « Cette annonce que j'obtiens de Dieu prend énormément de temps à restituer dans la rédaction en se fondant sur des références bibliques (ancien et nouveau testament). Car j'essaie non seulement de la rédiger d'une manière claire et simple afin de toucher tous les niveaux d'instructions, mais je fais en sorte de ne pas toucher aux convictions religieuses et des sectes religieuses chrétiennes pour que le message touche des membres de différentes appartenances confessionnelles dans tout le Liban et dans d'autres pays comme l'Égypte, la Syrie, la Jordanie. Aussi,j'envoie cette prière à des prêtres catholiques et orthodoxes, à des pasteurs, à des musulmans chiites et sunnites ainsi qu'à des athées. »

Son but est de prêcher car " le salut des âmes est pour tous ». Ses prières ne sont pas pensées comme contenant des convictions religieuses mais comme ayant avant tout pour objectif « de toucher les cœurs et [de] changer les gens ». À la question de savoir pourquoi la diffusion est faite par une liste sur WhatsApp et non par un groupe dédié, Pierre répond en évoquant un mode d'évangélisation singulier, de personne à personne : « quand j'envoie la prière la personne la reçoit de manière individuelle, cela ouvre une porte pour communiquer directement avec la personne afin de prêcher selon chaque cas. Je pense que les gens peuvent être timides dans un groupe et la communication n'aboutit pas à une conversation directe qui peut convaincre et répondre aux questions de cette personne ».

\subsection{Prière : entre l'auctorialité et la voix de Dieu}

Nous voyons donc émerger une diversité dans la pratique de partage de prières dans les groupes WhatsApp. Pierre, marqué par la spi- 
ritualité charismatique, se vit en communication avec l'Esprit Saint. D'après l'anthropologue américaine T.M. Luhrmann, « les chrétiens utilisent souvent le mouvement de leurs propres pensées pour juger que Dieu a parlé » (Luhrmann, 2017, p. 127). Pour les chrétiens, l'accent est mis en particulier sur la façon dont Dieu a communiqué avec le monde en la personne de Jésus de Nazareth, mais l'Esprit Saint est également compris comme inspirant, responsabilisant et communiquant avec les sujets de diverses façons, y compris par la prophétie. Dans certaines traditions chrétiennes, telles que le pentecôtisme et le mouvement charismatique œcuménique, l'accent est mis en particulier sur l'action expérimentée et permanente de l'Esprit Saint (Dein \& Cook, 2014).

Selon Pierre, l'Esprit Saint ou le Seigneur lui donnent des idées pour créer une prière, raison pour laquelle il prend beaucoup de temps pour la concevoir après avoir prié et écouté l'Esprit Saint, autrement dit, la voix de Dieu. Tandis que le prêtre maronite, nous l'avons vu, crée des prières assez rapidement et n'apprécie pas leur reprise anonymisée par un tiers. Sylvie, pour sa part, reprend des prières conçues par d'autres personnes ou des instituts et les modifie, en supprimant la marque auctoriale et toute référence à la prière originale. Sans doute les prières signées peuvent-elle être reprises et modifiées sans que celui qui les diffuse ait peur de la justice, car la véritable justice pour le croyant, c'est, souvent Dieu lui-même. Ceci explique sans doute que dans ce cas il n'y avait pas de problème de droits d'auteur et de propriété intellectuelle mais seulement d'auctorialité symbolique, d'autant que les histoires qui nourrissent l'esprit de ces prières appartiennent au domaine public, si l'on peut dire.

\section{Autorité du laïc et rôle du prêtre}

Un autre aspect qui émerge à l'observation de ces groupes WhatsApp chrétiens libanais est, au-delà de la question de l'auctorialité, la question des enjeux de pouvoir, notamment entre clercs et laïcs, qui, au-delà de la question de la propriété intellectuelle et de l'appropriation des ressources, traversent ces groupes. Nous pouvons voir notamment ces tensions à l'œuvre au sein de groupes animés par le prêtre Jean.

Les icônes de tous les groupes créés par le prêtre maronite Jean sont le plus souvent des photos de lui dans son église, car selon lui ces groupes lui appartiennent et il en est le gérant. « Ces groupes sont à moi, je suis le créateur et l'animateur de ces groupes, et chaque personne qui rejoint mes groupes pourra savoir que ces groupes sont animés par un 
prêtre et cela rend le groupe et les informations plus fiables aux yeux des membres du groupe ». La manière dont le prêtre expose ses photos en train de célébrer la messe comme icône du groupe atteste de l'intention d'exercer une forme de pouvoir symbolique et de contrôle spirituel et religieux dans le groupe.

\subsection{Groupe WhatsApp restreint}

Un groupe sur WhatsApp peut avoir plusieurs administrateurs qui peuvent ajouter et supprimer des membres. Dans un groupe, tout le monde peut intervenir sauf si le groupe est « fermé » (contribution restreinte), dans ce cas seuls les administrateurs peuvent écrire et envoyer des informations. Ce n'est qu'à partir de juin 2018 que WhatsApp a ajouté un nouveau paramètre de groupe par lequel seuls les administrateurs peuvent envoyer des messages à un groupe. C'est un moyen pour les usagers d'utiliser les groupes afin de recevoir simplement des annonces et des informations importantes. Le but de WhatsApp, en ajoutant ce paramètre, et en proposant le nouveau compte Business, est certainement d'encourager les entreprises à promouvoir et à créer des groupes WhatsApp comme moyen de communication one to many (et non many to many).

\subsection{Le groupe d'une personne lä̈que}

À cet égard, le groupe catholique créé par Léna est fermé : les membres ne sont que des lecteurs et des récepteurs de l'information. Les communications interactives restent donc restreintes dans ce groupe. Léna est aidée par une assistante dont le rôle est de parler en privé avec chaque personne qui quitte le groupe afin de connaître ses motivations, et en fonction de ses motivations, l'assistante tentera de la convaincre de réintégrer le groupe.

Avant juin 2018, les groupes n'étaient pas fermés, aussi Léna rappelait les règles de fonctionnement via le groupe WhatsApp afin de garder le contrôle sur les messages postés. Lorsqu'une personne ne respectait pas les règles d'appartenance au groupe, Léna l'excluait du groupe, et son assistante contactait cette dernière pour clarifier les raisons de l'exclusion. D'ailleurs, personne ne peut commenter ou poser des questions dans le groupe. Cependant, les questions pourront être posées en message privé à l'administratrice, qui n'a pas souvent le temps d'y répondre. De ce fait, plusieurs personnes ont été exclues du 
groupe et parmi elles le prêtre maronite Jean, qui est très présent sur les réseaux sociaux (chaîne YouTube et différents groupes WhatsApp). Le père Jean, qui possède lui aussi ses propres groupes, entre dans les autres groupes et diffuse ses prières car selon lui : " Il faut diffuser mes prières ainsi que les liens pour rejoindre mes groupes afin que les gens entrent dans mes groupes et agrandissent la communauté. » Lors de l'entretien que nous menons avec lui, il nous précise que "Léna ne respecte personne et n'a pas respecté mon statut de prêtre ! Elle se défend par son accompagnateur religieux mais cela ne lui donne pas le droit de manquer de respect envers un prêtre. Elle ne veut pas que j'entraîne des personnes de son groupe vers mes groupes... ». Au fur et à mesure, il a pu rencontrer des gens de son groupe et il leur a confié des responsabilités différentes, comme par exemple, la diffusion des prières d'un groupe à l'autre, la diffusion du culte sur sa chaîne YouTube, et la réponse aux questions posées par certains membres dans les groupes. Un enjeu d'audience et de gestion des publics caractérise donc ces groupes.

\subsection{Autorité dans un groupe en ligne}

Cette question des régimes d'autorité dans les groupes conduit Heidi Campbell à reprendre la tripartition webérienne entre « trois types d'autorité légitime : la domination légale, traditionnelle et charismatique $^{8} \gg$ (Campbell, 2007, p. 1046). Si par exemple le groupe WhatsApp faisait partie d'une organisation ou d'une association chrétienne, les supérieurs hiérarchiques pourraient former une autorité institutionnelle. L'autorité ou la domination d'un créateur et animateur d'un groupe religieux en ligne diffèrent en effet de la domination légale. Cependant, si l'action d'exclure le prêtre d'un groupe constitue un manque de respect par rapport à la figure du prêtre, l'autorité change de camp. Ainsi, l'autorité est détenue par le croyant administrateur qui a créé le groupe et dont les membres acceptent les conditions d'appartenance à celui-ci. En effet, les assistants dans les groupes sont des personnes de confiance que les créateurs ont choisies. Nous rencontrons donc une domination dans les groupes par le truchement de supérieurs hiérarchiques.

8 Notre traduction de l'anglais. 


\subsection{Autorité religieuse et laïque}

Pour les prêtres, « l'autorité religieuse naît de la détention d'un charisme » (Béraud, 2007). Ainsi, « le charisme de fonction du prêtre doit se trouver "rechargé" par différents processus : appel à la tradition, insistance sur la compétence, mise en avant des qualités voire des performances individuelles » (Béraud, 2007, p. 271). Si Weber (2014) souligne la nécessité de distinguer entre différentes formes d'autorité, « il convient de noter que l'autorité religieuse diffère du concept général d'autorité, en ce qu'elle s'appuie sur une forme particulière de légitimation, souvent liée à une source divine ${ }^{9} »$ (Campbell, 2007, p. 1046). Ainsi « la Grande Église ne se construit pourtant pas sans s'appuyer sur une autorité supérieure, mais c'est la tradition qui joue ce rôle » (Romanacce, 2013, p. 520). De par son statut de prêtre, Jean a cru pouvoir exercer une certaine forme d'autoritarisme avec Léna, mais ce fut sans effet. En outre, l'autorité de la réalité théologico-ecclésiologique ne s'est pas appliquée dans le réseau numérique. Si « c'est l'autorité dans sa dimension d'institution ou d'organe qui est introduite dans la définition de la réalité théologique, qu'il s'agisse du dogme, de la tradition ou, plus fondamentalement, de l'Église » (Chiron, 2006, p. 32), est-ce l'autorité institutionnelle qui est réduite d'un tel groupe WhatsApp ? Probablement. La fonction d'un prêtre dans le réseau WhatsApp du groupe de Léna est (seulement) de contrôler les prières et les informations envoyées. Et souvent, nous remarquons que dans certains groupes gérés par des laïcs, un prêtre observe les conversations et les données du groupe. C'est donc le laïc qui est devenu la personne qui évangélise et le prêtre a plutôt une fonction de « contrôle » sur le groupe : de cette façon, l'autorité institutionnelle s'en trouve réduite.

\subsection{Entre pouvoir et autorité religieuse}

«Les recherches portant sur le lien entre la religion en ligne et hors ligne ont souvent fait référence à des questions de pouvoir, ou en ont déduit que l'autorité et le contrôle sont des sujets de préoccupation pour les groupes religieux, affirmant que les pratiques en ligne remettent inévitablement en question la religion traditionnelle, tant au niveau des croyances que des pratiques ${ }^{10}$ ", fait observer Heidi

9 Notre traduction de l'anglais.

10 Notre traduction de l'anglais. 
Campbell (2007 p. 1046). Autrement dit, dans ces groupes, les membres surveillent l'administrateur et ses diffusions. L'administrateur est pensé comme astreint à l'exemplarité parce qu'il exerce une autorité dans son groupe. Si le prêtre s'occupe de sa communauté dans sa paroisse, des laïcs s'occupent de leur communauté en ligne : nous voyons bien le glissement de la communauté et de l'ecclésiologique institutionnel au techno-discursif et relationnel, l'un pouvant intégrer l'autre, qui lui échappe cependant, notamment par des conflits d'autorité. Ainsi, « les relations religieuses en ligne et hors ligne sont des tendances relatives au relâchement des frontières et des affiliations traditionnelles qui ont été observées et documentées par de nombreux sociologues de la religion $^{11} \gg($ Campbell, 2012, p. 21).

\section{Conclusion}

Dans le monde arabe et surtout au Liban, le réseau numérique WhatsApp est devenu progressivement un espace où la présence religieuse en ligne augmente, ouvrant un nouveau domaine d'études interdisciplinaires. En outre, bien que la religion catholique soit le socle commun parmi ces cinq acteurs du religieux numérique (Léna, Sylvie, Nabil, Pierre et le prêtre Jean), la pratique de la religion en ligne diffère selon la croyance et la culture de chaque croyant. Pour certaines, le groupe peut être considérer comme étant une Église ou un lieu sacré. Pour les autres, le groupe est simplement un lieu de partage de prières.

Qui plus est, selon notre traduction de l'anglais, « la médiatisation a également permis aux chercheurs d'expliquer les changements dans les formes traditionnelles d'autorité religieuse » (Campbell, 2016, p. 19). Ainsi, la pratique de l'autorité est présente dans certains groupes, et elle est appliquée au-delà des personnes religieuses, comme les prêtres, parce qu'avant tout, les règles du groupe sont plus importantes que l'autorité du religieux. La médiatisation se produit donc lorsque « les institutions médiatiques dominent l'ordre social, prenant la place autrefois occupée par les institutions religieuses en tant que pourvoyeurs et interprètes de la signification culturelle ${ }^{12} \gg$ (Campbell, 2016, p. 18). Dans ce cas, la pratique de la religion numérique a pondéré sinon dévalué la place de la hiérarchie de l'Église. Ainsi, selon notre traduction de l'anglais de Campbell (2012, p. 3) « les premières études ont souvent suggéré

11 Notre traduction de l'anglais.

12 Notre traduction de l'anglais. 
que l'utilisation d'Internet à des fins religieuses pourrait transformer la pratique et l'idéologie religieuse de manière révolutionnaire, en passant par la remise en question du rôle des autorités religieuses traditionnelles à la modification des attentes religieuses en matière de communauté et des liens ». Prêcher sur les réseaux numériques, et y entretenir une identité, devient le fait aussi bien des laïcs que des prêtres et des religieux. Enfin, l'espace de prière partagé devient comme un espace sacré, synonyme également, pour le Liban, de paix.

\section{Références}

Béraud, C. (2007). Le prêtre : personnage encore incontournable ou progressivement évincé ? Dans C. Béraud (dir.), Prêtres, diacres, laïcs : Révolution silencieuse dans le catholicisme français (pp. 245-273). Paris : PUF. Disponible à : https://www. cairn.info/pretres-diacres-laics--9782130557166-page-245.htm

Boëdec, F. (2006). Chrétiens d'Orient : Doutes et angoisses. Études, 405(11), 496-506. Doi : https://doi.org/10.3917/etu.055.0496

Bratosin, S., Mihaela, A. T. \& Iacob, C. (2010). La pratique du sacré dans le world wide web : une expérience innovante de la norme. Sciences de la société, 81, 121-134. Doi : https://doi.org/10.4000/sds.875

Brix, A. (2014). Lieux sacrés et espace ecclésial ( $\mathrm{IX}^{\mathrm{e}}-\mathrm{XV}^{\mathrm{e}}$ siècle). Cahiers de Fanjeaux, 46, Toulouse, Privat, 2011. Histoire urbaine, 39(1), 172-174. Doi : https://doi. org/10.3917/rhu.039.0172.

Campbell, H. (2007). Who's Got the Power? Religious Authority and the Internet. Journal of Computer-Mediated Communication, 12(3), 1043-1062. Doi : https:// doi.org/10.1111/j.1083-6101.2007.00362.x

Campbell, H. (2012). Understanding the Relationship between Religion Online and Offline. Journal of the American Academy of Religion. Disponible à : https://www. researchgate.net/profile/Heidi_Campbell5

Campbell, H. (2016). Surveying Theoretical Approaches within Digital Religion Studies. Disponible à : https://www.researchgate.net/profile/Heidi_Campbell5

Campbell, H. \& Teusner, P. (2011). Virtual Lives: Christian Reflection. Baylor University Press. Disponible à : https://www.researchgate.net/profile/Heidi_Campbell5

Catellani, A. (2014) Prier en ligne à partir d'images : observations sémiotiques sur le site Notre Dame du Web, MEI (Médiation et Information), 38, 101-112. Disponible à : https://mei-info.com/wp-content/uploads/2014/10/MEI38-101-111-Prier-enligne-a-partir-d-images.pdf

Chernet, D. (2008). Des règles ou des consignes ? Actualités en analyse transactionnelle, 127(3), 72-73. Doi : https://doi.org/10.3917/aatc.127.0072

Chidester, D. \& Linenthal, E.T. (1995). American Sacred Space. Bloomington, IN: Indiana University Press.

Chiron, J. (2006). « Une barrière éternelle. » L'autorité de l'Église dans la définition du dogme au XIX ${ }^{\mathrm{e}}$ siècle. Recherches de Science Religieuse, 94(1), 29-52. Doi : https:// doi.org/10.3917/rsr.061.0029 
Corm, G. (2008). Où en est la présence chrétienne en Orient ? Confluences Méditerranée, 3(3), 155-177. Doi : https://doi.org/10.3917/come.066.0155

De Witte, M. (2011). Touched by the Spirit: Converting the Senses in a Ghanaian Charismatic Church, Ethnos, 76(4), 489-509. Doi: https://doi.org/10.1080/00141844.20 11.620711

Dein, S. \& Cook, C.C.H. (2015). God Put a Thought into My Mind: The Charismatic Christian Experience of Receiving Communications from God. Mental Health, Religion \& Culture, 18(2), 97-113. Doi : https://doi.org/10.1080/13674676.2014.100 2761

Douyère, D. (2011). La prière assistée par ordinateur. Médium, 27(2), 140-154. Doi : https://doi.org/10.3917/mediu.027.0140

Douyère, D. (2014). L'image de piété chrétienne, objet-support de la croyance ? Communiquer la foi par l'image, de l'imprimé au numérique. Recherches en communication, 38, 29-46. Doi : https://doi.org/10.14428/rec.v38i38.50223

Douyère, D. (2015). De la mobilisation de la communication numérique par les religions. tic\&société, 9(1-2). doi : https://doi.org/10.4000/ticetsociete.1822

Douyère, D. (2016). Le christianisme en communication(s). Communication \& langages, 189. Doi : https://doi.org/10.4074/S0336150016013028

Favret-Saada, J. (2017). Sensibilités religieuses blessées. Christianismes, blasphèmes et cinéma 1965-1988. Gradhiva, 26, 230-232. Doi : https://doi.org/10.4000/gradhiva.3493

Forestier, L. \& Waymel, D. (2018). Les laïcs dans l'Église aujourd'hui : Benoît ou François ?. Nouvelle revue théologique, 140(4), 554-571. Doi : https://doi.org/0.3917/ nrt.404.0554

Jonveaux, I. (2013). Dieu en ligne. Expériences et pratiques religieuses sur Internet, Paris : Bayard, $248 \mathrm{p}$.

Jreijiry, R. (2018). L'image religieuse dans l'espace public libanais. Hermès, La Revue, 3(3), 53-59. Doi : https://doi.org/10.3917/herm.082.0053

Lapierre, J., Padis, M. \& Schlegel., J. (2015). Les images chrétiennes : inspirations et survivances. Esprit, 2, 129-134. doi : https://doi.org/10.3917/espri.1502.0129

Le Crosnier, H. (2014). Internet et numérique. Hermès, La Revue, 70(3), 25-33. Doi : https://doi.org/10.3917/herm.070.0023

Luhrmann, T. M. (2017). Knowing God. The Cambridge Journal of Anthropology, 35(2), 125-142. Doi : https://doi.org/10.3167/cja.2017.350210

Romanacce, F. (2013). Église et Églises : réflexion sur les questions d'autorité dans les communautés chrétiennes au $\mathrm{II}^{\mathrm{e}}$ siècle. Recherches de Science Religieuse, 101(4), 517-527. Doi : https://doi.org/10.3917/rsr.134.0517

Salatko, G. (2016). Le Dieu situé. Une enquête sur la fabrique de l'art sacré dans le catholicisme contemporain. Paris : L'Harmattan.

Weber, M. (2014). Les trois types purs de la domination légitime (Traduction d'Elisabeth Kauffmann). Sociologie, 5(3), 291-302. Doi : https://doi.org/10.3917/socio.053.0291

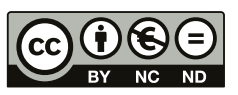

«Attibution - pas d'utilisation Commerciale - Pas de Modification 4.0. International» (CC BY-NC-ND) 